\title{
Résistences interieures. Visages du conflit dans le journal personnel, S. Lannegrand et V. Montémont (éds.)
}

\section{Luana Doni}

\section{(2) OpenEdition}

Journals

\section{Edizione digitale}

URL: http://journals.openedition.org/studifrancesi/10097

DOI: $10.4000 /$ studifrancesi. 10097

ISSN: 2421-5856

\section{Editore}

Rosenberg \& Sellier

\section{Edizione cartacea}

Data di pubblicazione: 1 août 2017

Paginazione: 418-420

ISSN: 0039-2944

\section{Notizia bibliografica digitale}

Luana Doni, «Résistences interieures. Visages du conflit dans le journal personnel, S. Lannegrand et V. Montémont (éds.)», Studi Francesi [Online], 182 (LXI | II) | 2017, online dal 01 août 2017, consultato il 07 janvier 2021. URL: http://journals.openedition.org/studifrancesi/10097 ; DOI: https://doi.org/10.4000/ studifrancesi. 10097

Questo documento è stato generato automaticamente il 7 janvier 2021.

\section{cc) (†) $\odot$}

Studi Francesi è distribuita con Licenza Creative Commons Attribuzione - Non commerciale - Non opere derivate 4.0 Internazionale. 


\title{
Résistences interieures. Visages du conflit dans le journal personnel, $\mathrm{S}$. Lannegrand et V. Montémont (éds.)
}

\author{
Luana Doni
}

\section{NOTIZIA}

Résistences interieures. Visages du conflit dans le journal personnel, études réunies et présentées par Sylvie LANNEGRAND et Véronique MONTÉMONT, Louvain-la-neuve, Éditions Academia, 2016, «Au Cœur Des Textes» 32, 176 pp.

1 Il volume raccoglie quelle testimonianze che hanno trovato poco spazio nella storia della letteratura intima ma che si rivelano fondamentali in quanto resoconto di una resistenza interiore portata avanti dall'individuo di fronte alla situazione storicosociale che lo vede protagonista nel tempo della scrittura. Il fulcro intorno al quale si articola la presente indagine portata avanti dal programma di ricerca franco-irlandese Ulysses, è lo studio del diario intimo tra il xIx e il xx secolo con particolare attenzione alle prospettive legate alla sfera politica, sociale e sessuale.

La prima sezione della raccolta, intitolata «Face à la guerre», è consacrata alla riflessione sui diari tenuti durante i due grandi conflitti mondiali.

Nel contributo di Marion KRAUTHAKER (Le journal d'un voyageur pendant la guerre de George Sand ou le parcours d'une femme-citoyen, pp. 13-26), l'esperienza di George Sand di fronte alle drammatiche svolte della guerra franco-prussiana. Fatto oggetto di studio superficiale da parte degli storici della letteratura, il Journal d'un voyageur pendant la guerre tenuto da G. Sand tra il 1870 e il 1871, tiene conto del difficile cammino intrapreso dall'autrice per conciliare l'attività politica con quella domestica. Generalmente avvezza all'utilizzo della scrittura epistolare, George Sand comincia a tenere un diario all'indomani della sconfitta di Sedan, nella preoccupazione per le sorti della Francia. Attraverso espedienti stilistici che denotano una probabile volontà di 
pubblicazione del diario, come nota David Powell, la diarista si pronuncia a favore di una vera repubblica socialista non risparmiando il proprio malcontento nei confronti di quelle politiche sostenitrici della guerra ad oltranza. Nonostante il titolo dell'opera tradisca gli intenti dell'autrice, George Sand si esprime in quanto voce femminile facendo così del diario un pretesto per la riconciliazione tra il suo impegno politico $\mathrm{e}$ letterario e la sua posizione di donna e venendo a infrangere la solidificata immagine della Bonne Dame de Nohant che la caratterizzò gli ultimi anni della sua vita.

L'indagine di Marion Krauthaker si sposta in seguito verso l'Irlanda della prima Grande Guerra (La souffrance entre les lignes: le journal de Mary Martin, mère irlandaise sous la Première Guerre mondiale, pp. 27-40) e in particolar modo sulla figura di Mary Aloysius Martin. Il diario di Mary Martin è datato 1916, anno durante il quale l'autrice non ha più notizie del figlio Charlie partito per il fronte nel 1915. Non destinato alla pubblicazione, il diario diventa un dialogo immaginario tra una madre e suo figlio scomparso interpolato dalla preziosa testimonianza della vita di una donna irlandese in tempo di guerra. Le attività quotidiane, relegate soprattutto alla sfera domestica, non sono che una copertura che cela i veri intenti della scrittrice ovvero le ricerche per trovare il figlio Charlie ancora in vita. Un modo per tenere accesa la speranza o più semplicemente per cullare dentro di sé una menzogna che le permetta di continuare a vivere fino al punto in cui l'accettazione di una realtà lascia il posto al silenzio e all'interruzione di un diario che diventa muto compianto.

Storie di uomini e donne civili nel saggio di Françoise SIMONET-TENANT (Mai-juin 1940 dans les journaux personnels, pp. 41-54), che analizza testi di diaristi improvvisati dall'esigenza di raccontare un'esistenza sconvolta dalla guerra. Quattordici frammenti di diario studiati con l'intento di capire se le tracce lasciate dalle testimonianze possano contribuire in parte alla scrittura della Storia. Tra i contributi, quello di Edith Thomas sull'esodo da Parigi ad Arcachon vissuto come la privazione della vita cui si era abituati, e quelli di Reine Juliette Klavatz e di Paul Léautaud che invece non hanno abbandonato la loro città ridotta ormai alla stregua di un deserto. L'angoscia generata dall'impotenza nei confronti dell'occupazione tedesca è testimoniata nei diari di Jean Guéhenno, André Gide e Simone de Beauvoir. La scrittura quotidiana diventa un modo di resistere alla devastazione del vissuto, un modo di sperare e di affermarsi, di umanizzare la memoria collettiva per ritrovare ciò che non ha lasciato alcuna traccia negli archivi istituzionali.

6 Poca circolazione è stata destinata a un altro contributo sul vissuto soggettivo della Guerra Mondiale, quello di Micheline Bood e del suo diario Les années doubles presentato nel contributo di Catherine VIOLLET («Nous n'aurons plus jamais notre âme de ce soir» Micheline Bood, Journaux 1939-1947, pp. 55-67). Pubblicato parzialmente nel 1974, il diario alterna problematiche riguardanti la vita in tempo di guerra e il quotidiano di una giovane donna al termine degli studi. La riflessione si concentra pertanto su tre aspetti della vita e della scrittura di Micheline Bood: il problema del cibo durante la guerra, gli atti di resistenza all'occupazione che denotano il solido patriottismo della diarista, e infine il risveglio della sessualità della giovane Micheline suscitato dagli ufficiali tedeschi. Verso la fine dell'occupazione il diario smette di diventare un sussidio alla resistenza interiore per assumere i contorni di un racconto di formazione dell'autrice che si appresta a entrare nel mondo del lavoro.

7 Nel vivo della questione delle leggi razziali durante la Seconda Guerra Mondiale entra la relazione di Cyril GRANGE (Le journal de Christian Lazard sous l'Occupation: exemple du dilemme entre rester et partir, pp. 69-85) in cui a prevalere è il dilemma di un uomo diviso 
tra la volontà di restare fedele alla propria patria e la necessità di partire nella speranza di salvarsi. Quello di Christian Lazard, ebreo francese figlio di Simon Lazard, uno dei fondatori della celebre Banque Lazard, non è esattamente un diario intimo quanto piuttosto l'agenda au jour le jour di una vita che incrocia nel suo inesorabile scorrere le vicissitudini del dramma economico-politico in corso. Sposato con Annette May di religione cattolica, le riflessioni di Lazard sul suo porsi dinanzi al conflitto in quanto ebreo sono ispirate dalla convinzione che sia la nazionalità a fare l'uomo e non il suo orientamento religioso. Pertanto, il dilemma che lo aveva portato nel 1938 a tenere il diario si risolve nella volontà dell'autore di rendere testimonianza d'amore nei confronti della patria e di resistere, nonostante le insistenze di amici e conoscenti, rivendicando il diritto di restare.

8 Sempre sul versante dei diari concentrati sull'esperienza della segregazione razziale, il diario di Hélène Berr mette in scena la vita di una giovane studentessa della Sorbona prima e dopo la promulgazione della leggi anti-ebraiche. Véronique MONTÉMONT ( Le journal d'Hélène Berr, pp. 87-103), nella riflessione sulle particolarità del diario tenuto dalla giovane durante gli ultimi anni dell'occupazione, mostra come, nella scrittura di Hélène Berr, l'importanza dello sguardo che testimonia l'evidenza di un problema e lo scatenarsi della brutale percezione della discriminazione, funga da acceleratore di sentimenti: esperire il possibile prima che diventi troppo tardi. Nel vortice sensoriale in cui la giovane sembra immersa, non manca tuttavia la parte dedicata al disprezzo per la politica di Pétain e per le misure del regime di Vichy che fa del diario un vero e proprio reportage sulla situazione delle vittime al fine di preservare una traccia di ciò che è stato.

La seconda sezione della raccolta intitolata «Lutter pour être soi» è dedicata al diario in quanto espressione di un dissidio interiore vissuto dal soggetto scrivente. Il primo contributo a questo proposito riguarda il diario di Adèle Hugo raccontato dalle parole di Jean-Marc HOVASSE (Trois amours d'Adèle H., pp. 107-124). La riflessione proposta si concentra sull'analisi delle sezioni del diario concernenti gli amori importanti della figlia più giovane di Victor Hugo (quello per Auguste Vacquerie, quello per l'artista Auguste Clésinger e quello per John Rose), sottolineando la forma con cui la giovane espone i suoi più intimi sentimenti attraverso la scrittura. L'alternanza di passaggi dai toni lirici a quelli dalla forma gergale e pressoché incomprensibile denotano la particolarità dello stile dell'autrice che viene spiegata da Hovasse come segno della voglia di contrapporsi all'immagine di un padre dalla fama ingombrante $o$, più semplicemente, come tentativo di prendere le distanze da un prevedibile destino che la vede rilegata al semplice compito di moglie e di madre.

Un destino che risulta troppo stretto per alcune donne tra le quali Hélène Hoppenot che sposa, invece, lo è stata. Nel saggio di Hélène GESTERN (Les itinéraires d'une femme libre: Hélène Hoppenot, pp. 125-138) è analizzato il diario di una delle pioniere della libertà femminile, diario tenuto lungo sessant'anni, resoconto di una vita semi-nomade stimolata dalla voglia di conoscenza. L'aspetto caratteristico del diario di Hélène Hoppenot è questo suo non celarsi dietro al rigore dell'epoca, non far maschera della propria libertà. Benché le parti relative alla sfera privata siano pressoché assenti, a parte quelle riguardanti lo stato d'animo generato dalla gravidanza di Hélène vissuta dalla stessa come un impedimento, non è invece dissimulata la posizione dell'autrice di fronte alla questione della donna nelle società patriarcali, del rancore nei confronti dei tedeschi e del suo sentirsi anticlericale. Questa volontà di impegno sociale avvicina il 
diario di Hélène Hoppenot a quello di un altro scrittore, lucidamente analizzato nella riflessione di Sylvie LANNEGRAND (De la cause homosexuelle au scandale de l'écriture: le journal de Yves Navarre, pp. 139-149): il diario di Yves Navarre. Nonostante l'opera di Navarre si accinga quasi a congedarsi nei territori dell'oblio, il diario resta un contributo interessante dal punto di vista della sua molteplicità. Accanto alle riflessioni sulla scrittura, è presente una sezione dedicata al viaggio e alle considerazioni sulla società e i costumi degli anni Settanta che contiene un'ampia gamma di appunti sulla questione delle ingiustizie sociali ai danni del proletariato e sul dibattito riguardante la legittimità dell'omosessualità. Dibattito, questo, che rimane aperto anche nella relazione che chiude la seconda sezione, sempre ad opera di Sylvie LANNEGRAND (Jocelyne François: le corps à l'épreuve et l'engagement dans l'écriture, pp. 151-160). Due sono le dimensioni portate avanti dal diario di Jocelyne François pubblicato in tre volumi: una corporeo-sensuale, dal titolo Le Cahier vert, Journal 1961-1989 e un'altra, che comprende i diari dal 1990 al 2007, caratterizzata dalla scrittura vissuta come impegno esistenziale e atto di resistenza nei confronti di una società ritenuta troppo vincolante per la libertà dell'individuo. 